\title{
Fallacies of composition in nonlinear marketing models
}

\author{
Gian Italo Bischi $(*)$ \\ DESP - Department of Economics, Society, Politics \\ University of Urbino - Italy \\ email: gian.bischi@uniurb.it \\ Lorenzo Cerboni Baiardi \\ DiSBeF - Department of Base Sciences and Fundamentals \\ University of Urbino - Italy \\ email: lorenzo.cerboni@gmail.com
}

\begin{abstract}
In this paper we consider some nonlinear discrete-time dynamic models proposed in the literature to represent marketing competition, and we use these models to critically discuss the statement, often made in economic literature, that identical agents behave identically and quasi-identical ones behave in a similar way. We show, through examples and some general mathematical statements, that the one-dimensional model of a representative agent, whose dynamics summarize the common behaviour of identical interacting agents, may be misleading. In order to discuss these topics some simple methods for the study of local stability and bifurcations are employed, as well as numerical examples where some results taken from the literature on chaos synchronization are applied to two-dimensional marketing models that exhibit riddling, blowout and other global phenomena related to the existence of measure-theoretic attractors.
\end{abstract}

Key-words: Nonlinearity, synchronization, market share models, composition, representative agent.

(*) Corresponding author. Email:gian.bischi@uniurb.it 


\section{Introduction}

"The conventional assumption made in macroeconomic theory is that there are many identical agents, whose behaviour is summarized by that of the representative agent". This sentence, taken from [50], expresses a quite evident property provided that the economic agents are truly identical, i.e. characterized by the same features and identical starting conditions. Indeed, the concept of representative agent is widely used (even abused) in economic modelling (see e.g. [24], [29], [50], [26]). Moreover, a stream of literature exists where the common time evolution of $n$ identical agents is reduced to a one-dimensional dynamic model that summarizes the common aggregate behaviour of the $n$ agents, and their numerosity $n$ is taken as a bifurcation parameter, so that the relation between stability and the number of agents is studied (see e.g. [48] and [14] in oligopoly theory, [22] in marketing modelling). This is a classical issue in ecological modelling as well, where the trade-off between the number of species and ecosystem stability is often considered (see e.g. [35]). Analogously, in economics the relation between market stability and the number of firms producing homogeneous goods is often considered.

However, it's quite evident that the assumption of agents with absolutely identical features and identical starting conditions is not generic, we may say a zero probability event, as in real systems one can at most say that agents are practically identical, or almost identical or quite similar and so on. Of course, economists are aware of this difficulty, and in fact statements like the following are very common (we quote again [50]) "although simple examples can be constructed wherein the preferences of the aggregate might misrepresent those of diverse agents, it has not been established that this is a significant problem in the analysis of macroeconomic shocks. (...) Unless the agents have very different characteristics, it seems reasonable to expect that each individual will respond to aggregate macroeconomic shocks hitting the general economy in more or less the same qualitative way (though not necessarily identically), in which case the aggregate should behave in a qualitative similar way as well". In other words, unless the agents have very different characteristics, it seems reasonable to expect that each individual will behave in more or less the same qualitative way (though not necessarily identically) so that the behavior of the aggregate system is still sufficiently well summarized by the behavior of the representative agent. However, this point has been recently criticized by some authors ([29], [47], [2], [9]).

A more formal statement of the problem is the following. If the time evolution of an economic system with several interacting agents is represented by an $n$-dimensional dynamical system, when the agents are identical, i.e. they are characterized by identical parameters, then the dynamical system becomes symmetric in the sense that it remains the same by interchanging the agents. This symmetry property implies that an invariant one-dimensional subspace exists, corresponding to the obvious statement that identical agents, starting from identical initial conditions, behave identically for each time, i.e. if $n$ identical agents start from identical initial conditions, say $x_{1}(0)=x_{2}(0)=\ldots=x_{n}(0)$ then their dynamic behaviour will be characterized by $x_{1}(t)=x_{2}(t)=\ldots=x_{n}(t)$ for each $t \geq 0$. Such synchronized dynamics are governed by a one-dimensional dynamical system, given by the restriction of the n-dimensional system to the invariant subspace on which the synchronized dynamics occur, which can be seen as the model of a representative agent, whose dynamic behavior summarizes the common behavior of the identical agents (see e.g. [9], [31]).

The main goal of the paper is to show, through some examples and some general mathematical statements, that the one-dimensional restriction may be quite misleading. In order to support this claim we use two kinds of arguments. In the first part of the paper we show some examples, taken from the literature, where, due to algebraic simplifications or mathematical cancellations along the invariant submanifold, the dynamic properties of the one-dimensional system may be completely different from those of the complete model. For example, as we shall see, highly nonlinear models with very complicated dynamic behaviours may dramatically collapse into a trivial linear dynamic model when identical agents are considered, or models based on similar kinds of dynamic interactions, which exhibit similar dynamic behaviours, give rise to quite different kinds of dynamic qualitative behaviours when the assumption of identical agents is imposed and the corresponding restrictions are considered.

In the second part of the paper we investigate the question of the effects of small heterogeneities, i.e. small deviations from the condition of identical agents, on the basis of a stream of literature on symmetric 
dynamical systems (see [17] and references therein). Indeed, even in the case of identical agents, if their time paths start from slightly different initial conditions they may not synchronize in the long run, so that their asymptotic behaviors become very different from the one expected according to the model of representative agent. Moreover, a slight modification of the parameters with respect to the symmetric situation, may lead to a qualitatively different dynamic evolution (see e.g. [44], [40], [52], [32]). In other words, the destruction of the invariant submanifold, on which synchronized dynamics takes place, implies that the attractors that characterize the long-run behavior of the one-dimensional model of the representative agent are substituted by new attractors that may be very different from those existing when symmetry is present.

Even if the examples considered in this paper are taken from the literature on dynamic marketing models, the results obtained can be applied to a broad class of multi-agent dynamic models in economics and social sciences with interacting agents, as well as in ecological models of interacting species sharing the same (or similar) ecological niche, or more generally in physical and engineering systems where identical or almost identical coupled nonlinear oscillators are represented.

The plan of the paper is as follows. In Section 2 we consider three different kinds of adaptive adjustment models, proposed in the literature to represent market share attraction of firms which compete in the market, and we show that even if the models with heterogeneous interacting firms exhibit similar properties, in the symmetric case of identical firms starting from identical initial conditions, whose dynamics are governed by the restrictions to invariant subspace where synchronized dynamics occur, the dynamic properties are quite different and in some cases even misleading. In Section 3 we focus on two of the dynamic marketing models examined in section 2, one proposed in [22] and one proposed in [7] (see also [8], [31]) with two interacting firms, and we analyze what happens in the case of identical firms starting from slightly different initial conditions as well as in the case of slight differences in one parameter. Section 4 concludes. Some details on the study of local stability and bifurcations of the steady states of models considered in the paper are given in the Appendix A, whereas in the Appendix B a short overview of some recent results about chaos synchronization and related phenomena is reported in order to make the paper more self-contained.

\section{Some examples of representative firm dynamics in marketing mod- els}

The different dynamic models described in this section belong to the class of market share attraction models, a well established stream in economic literature (see e.g. [6], [18], [20], [7], [22]) based on the following assumptions.

Let us consider $n$ firms that sell homogeneous goods in a market with sales potential $B$ in terms of customers' market expenditures (also denoted as market contribution $M C$ by some authors, see e.g. [22]) and let $A_{i}(t)$, $i=1, \ldots, n$, denote the attraction of customers to firm $i$ at time period $t$, where $t \in \mathbb{N}$ represents an event-driven discrete time variable. The key assumption in marketing literature is that the market share for firm $i$ at time $t$ is given by

$$
s_{i}(t)=\frac{A_{i}(t)}{\sum_{j=1}^{n} A_{j}(t)}
$$

If $x_{i}$ denotes marketing spending of firm $i$, following [20], see also [22], we assume that attraction is given by

$$
A_{i}=a_{i} x_{i}^{\beta_{i}}
$$

where the positive constants $a_{i}$ denote the relative effectiveness of effort expended by the firm $i$ and the parameters $\beta_{i}$ denote the elasticity of the attraction of firm (or brand) $i$ with regard to the marketing effort, as $\frac{d A_{i}}{d x_{i}} \frac{x_{i}}{A_{i}}=\beta_{i}$. On the basis of these assumptions, the one-period net profit of firm $i$ is given by

$$
\Pi_{i}(t)=B s_{i}(t)-x_{i}(t)=B \frac{a_{i} x_{i}^{\beta_{i}}(t)}{a_{i} x_{i}^{\beta_{i}}(t)+\sum_{j \neq i} a_{j} x_{j}^{\beta_{j}}(t)}-x_{i}(t)
$$


The models described in the following propose different adaptive methods used by firms to decide next period efforts, based on the profit function (2).

\subsection{The model with best reply}

Farris et al. [22] consider the case of unit elasticities $\beta_{i}=1, i=1, \ldots, n$, and assume that agents at each time $t$ decide next period spending $x_{i}(t+1)$ by solving the optimization problem $\max _{x_{i}} \Pi_{i}(t+1)$, where $\Pi_{i}$ is given by (2). From the first order conditions $\frac{\partial \Pi_{i}(t+1)}{\partial x_{i}}=0$ they get

$$
x_{i}(t+1)=\sqrt{B \frac{\sum_{j \neq i} a_{j} x_{j}^{(e)}(t+1)}{a_{i}}}-\sum_{j \neq i} a_{j} x_{j}^{(e)}(t+1)
$$

where $x_{j}^{(e)}(t+1)$ represent the expectation of firm $i$ about firm $j$ spending at time $t+1$ on the basis of the information set of firm $i$ at time $t$. Assuming naive expectations $x_{j}^{(e)}(t+1)=x_{j}(t)$ they get the dynamic model

$$
x_{i}(t+1)=R_{i}\left(\sum_{j \neq i} a_{j} x_{j}(t)\right)=\sqrt{B \frac{\sum_{j \neq i} a_{j} x_{j}(t)}{a_{i}}}-\sum_{j \neq i} a_{j} x_{j}(t)
$$

usually denoted as "Best Reply with naive expectations". Farris et al. then consider the symmetric case of identical players

$$
a_{i}=a \text { and } x_{i}(0)=x(0) \text { for each } i
$$

which implies that $x_{i}(t)=x(t)$ for each $t \geq 0$. In this case $\sum_{j \neq i} a_{j} x_{j}(t)=(n-1) a x(t)$ and the common dynamic behaviour of the identical firms is governed by the one-dimensional difference equation

$$
x(t+1)=f(x(t))=\sqrt{B(n-1) x(t)}-(n-1) a x(t) .
$$

Farris et al. [22] numerically show that (5) exhibits a bifurcation from stable equilibrium to stable periodic oscillations, and then chaotic dynamics, as the number $n$ of firms increases. Indeed, this statement can be analytically proved as follows. The first order derivative

$$
f^{\prime}(x)=\frac{\sqrt{B(n-1)}}{2 \sqrt{x}}-(n-1) a
$$

computed at the unique positive equilibrium

$$
x_{E}=\frac{B(n-1)}{(1+(n-1) a)^{2}}
$$

becomes $f^{\prime}\left(x_{E}\right)=\frac{1-(n-1) a}{2}$, and from the stability condition $-1<f^{\prime}\left(x_{E}\right)<1$, we get

$$
(n-1) a \leq 3 \text {, i.e. } n<1+\frac{3}{a}
$$

In the case of unit efficiency $a=1$, it follows that the marketing system loses stability when the number of firms exceeds 4 , expressed in [22] by the statement " 5 is a crowd", in the sense that 5 firms imply instability.

Farris et al. [22] also propose a different adjustment, known as adaptive adjustment towards best reply, see also [42], [30], [14]

$$
x_{i}(t+1)=\left(1-\lambda_{i}\right) x_{i}(t)+\lambda_{i} R_{i}\left(\sum_{j \neq i} a_{j} x_{j}(t)\right)
$$


where $i=1, \ldots, n$ and the constants $\lambda_{i} \in[0,1]$ represent the attitude of firm $i$ to adopt the best reply, whereas $\left(1-\lambda_{i}\right)$ is the anchoring attitude to maintain the previous spending decision, i.e. a measure of inertia. The model (8) is a generalization of (3) because it reduces to it for $\lambda_{i}=1, i=1, \ldots, n$, whereas it tends to absolute inertia of firm $i$, i.e. $x_{i}(t+1)=x_{i}(t)$, as $\lambda_{i} \rightarrow 0$. Moreover, the model (8) has the same equilibria as the best reply model (3), and under the assumption

$$
a_{i}=a, \lambda_{i}=\lambda \text { and } x_{i}(0)=x(0) \text { for each } i
$$

the one-dimensional model of the representative agent

$$
x(t+1)=(1-\lambda) x(t)+\lambda(\sqrt{B(n-1) x(t)}-(n-1) a x(t))
$$

ensures asymptotic stability of the positive equilibrium (6) provided that

$$
(n-1) a<\frac{4-\lambda}{\lambda} \text {, i.e. } n<1+\frac{4-\lambda}{\lambda a}
$$

hence the stability condition holds for an arbitrarily large number of firms provided that $\lambda$ is sufficiently small, i.e. under the assumption of inertia or "prudent behaviour" of the identical firms.

Of course, the dynamic behaviour of the complete $n$-dimensional marketing model, with heterogeneous firms due to differences in some parameters and/or in the initial conditions, is more rich. As an example we shown a numerical snapshots of dynamic scenarios of the model (8) with $n=2$ in Figure 1. A short analytical study of existence and local stability of the equilibrium points of (8) with $n=2$ is given in the Appendix A.

\section{Fig. 1 APPROX. HERE}

\section{$2.2 \quad$ A different kind of adjustment}

Following [7] and several other authors, see e.g. [6], [18], [19], we now consider the assumption that the two competitors change their marketing efforts adaptively in response to the profits achieved in the previous period. In particular, the marketing efforts in period $t+1$ are determined by

$$
x_{i}(t+1)=x_{i}(t)+\lambda_{i} x_{i}(t) \Pi_{i}(t)=x_{i}(t)+\lambda_{i} x_{i}(t)\left(B \frac{a_{i} x_{i}^{\beta_{i}}(t)}{\sum_{j=i}^{n} a_{j} x_{j}^{\beta_{j}}(t)}-x_{i}(t)\right)
$$

where (2) has been used. In this model the decision of the firms is driven by profits obtained in the previous period with a type of anchoring and adjustment heuristic widely used in decision theory (see e.g. [46], [53]). The parameters $\lambda_{i}>0$ measure the speed of adjustment.

Also in this case a wide spectrum of rich dynamic scenarios is obtained, see [7] for an extensive study of the case with $n=2$. Two exemplary cases are shown in Figure 2 (see appendix A for a brief analytical study of equilibrium stability).

\section{Fig. 2 APPROX. HERE}

We can say that the global dynamic properties of this model are similar to the ones observed in the profit optimization model of the previous subsection. However, under the assumption of identical firms starting from identical initial conditions the one-dimensional restriction of (10) to the invariant diagonal is

$$
x(t+1)=x(t)\left(1+\frac{\lambda B}{n}-\lambda x(t)\right)
$$


a quadratic map conjugate to the logistic map $z(t+1)=\mu z(t)(1-z(t))$ with $z=\frac{n \lambda}{n+\lambda B}$ and parameter $\mu=1+\frac{\lambda B}{n}$. Hence in this case the unique positive equilibrium $x_{E}=B / n$ is stable for

$$
1+\frac{\lambda B}{n} \leq 3 \text {, i.e. } n \geq \frac{\lambda B}{2}
$$

a result completely different from the one obtained by Farris et al. [22] and recalled above, being in this case the system stable for a sufficiently large number of firms.

\subsection{Gradient dynamics}

Let us consider here a dynamic adjustment based on profit gradient (or marginal profits) a decision rule often proposed in the economic literature on boundedly rational agents (see e.g. [3], [5], [51], [16], [15], [23], [43], [11], [10])

$$
x_{i}(t+1)=x_{i}(t)+\lambda_{i} x_{i}(t) \frac{\partial \Pi_{i}(t)}{\partial x_{i}}=x_{i}(t)+\lambda_{i}\left(B \beta_{i} \frac{a_{i} x_{i}^{\beta_{i}}(t) \sum_{j \neq i} a_{j} x_{j}^{\beta_{j}}(t)}{\left(\sum_{j=i}^{n} a_{j} x_{j}^{\beta_{j}}(t)\right)^{2}}-x_{i}(t)\right)
$$

where $\lambda_{i}>0, i=1, \ldots n$, is the speed of adjustment which measures how strongly agent $i$ reacts to signals of increasing or decreasing profits by increasing or decreasing marketing efforts respectively. The model (13) with $n=2$ has been studied in [10], where it is shown that a unique positive equilibrium exists that loses stability giving rise to oscillatory behaviours, see e.g. Figure 3 (and the corresponding analytic study of local stability in the Appendix A).

We consider again the case of $n$ identical players

$$
\lambda_{i}=\lambda, a_{i}=a, \beta_{i}=\beta \text { and } x_{i}(0)=x(0), i=1, \ldots, n
$$

and we compute the one-dimensional restriction that governs the corresponding dynamics of the representative agent

$$
x(t+1)=(1-\lambda) x(t)+\frac{B(n-1)}{n^{2} a x^{1-\beta}}
$$

Notice that in the case of unit elasticity $\beta=1$ (as assumed in [22]) it becomes a linear contractive onedimensional map, hence always globally asymptotically stable (independently of the number of firms $n$ ).

To sum up, in this Section we have considered three different $n$-dimensional dynamic marketing models with similar adaptive adjustment processes based on the same profit function, that exhibit similar dynamic behaviours (at least in the two-dimensional case). However, we have shown that they are characterized by completely different behaviours when their one-dimensional restriction governing the synchronized dynamics is considered.

Fig. 3 APPROX. HERE

\section{The problem of synchronization in the case of two firms}

In this section we study two of the dynamic marketing models presented in the previous section with $n=2$, and the two firms are assumed to be identical, i.e. characterized by the same parameters and starting from the same initial conditions, or quasi-identical, i.e. with a slight difference between the parameters and/or their initial conditions. A recent stream in the literature on applied mathematics shows that the symmetric models which derive from the assumption of identical coupled units (agents, oscillators etc.) exhibit, in many situations, non-generic dynamical behaviors. We first consider the case of two identical firms that start from different initial effort allocations, with the related question if the trajectories synchronize over time, i.e., if 
$\left\|x_{1}(t)-x_{2}(t)\right\| \rightarrow 0$ as $t \rightarrow+\infty$. In this case, the initial difference between the marketing efforts of the two firms, $\left\|x_{1}(0)-x_{2}(0)\right\|>0$, would cancel out within a reasonably short time span, so we can safely ignore the transient dynamics of the two-dimensional system, and consider the one-dimensional model of the representative firm instead. If synchronization takes very long or does not occur at all, then the concept of the representative firm becomes meaningless. This leads to the following question: under which conditions do the trajectories of identical competitors which start from different initial effort choices synchronize, and how does this depend on the difference of the initial efforts?

The second topic considered concerns the question if small heterogeneities between the two firms - a small mismatch of some of the parameters - matter for synchronization or not. Answering these questions is not easy, since new dynamic phenomena may appear, especially in the presence of chaotic behavior. In this case chaotic synchronization may occur, a phenomenon that has been extensively studied in the recent physical and mathematical literature. In order to make the paper more self-contained, in the Appendix B we give a short overview of some notions and results that can be found in this stream of literature, and we refer to [1], [4], [17], [52], [12], [8] and references therein for a deeper insight.

\subsection{Synchronization and synchronization failure in two marketing models}

Let us first consider model (10) with $n=2$ competitors.

$$
T:\left\{\begin{array}{l}
x_{1}(t+1)=x_{1}(t)+\lambda_{1} x_{1}(t)\left(B \frac{a_{1} x_{1}^{\beta_{1}}(t)}{a_{1} x_{1}^{\beta_{1}}(t)+a_{2} x_{2}^{\beta_{2}}(t)}-x_{1}(t)\right) \\
x_{2}(t+1)=x_{2}(t)+\lambda_{2} x_{2}(t)\left(B \frac{a_{2} x_{2}^{\beta_{2}}(t)}{a_{1} x_{1}^{\beta_{1}}(t)+a_{2} x_{2}^{\beta_{2}}(t)}-x_{2}(t)\right)
\end{array}\right.
$$

A general study of this map is given in [7], and its features in the symmetric case, i.e. identical competitors for which

$$
\lambda_{1}=\lambda_{2}:=\lambda ; \beta_{1}=\beta_{2}:=\beta ; a_{1}=a_{2}
$$

are considered in [8], where it is argued that the parameter $\beta$ measures the degree of competition between the firms. We now use this example to show the effects of small heterogeneities in the initial condition by using the methods described in the previous subsection. The restriction of the resulting symmetric map to the invariant diagonal $\Delta$ (see (43) in Appendix B) is given by

$$
T_{\Delta}(x)=\left(1+\frac{\lambda B}{2}\right) x-\lambda x^{2} .
$$

which can be rewritten as a standard logistic map $z^{\prime}=\mu z(1-z)$ throw the linear transformation $x=$ $\frac{z}{\lambda}\left(1+\frac{\lambda B}{2}\right)$, where

$$
\mu=1+\frac{1}{2} \lambda B
$$

For the symmetric map, the Jacobian matrix, computed at a point of the diagonal $\Delta$ results

$$
\boldsymbol{J}=\left(\begin{array}{cc}
1-2 \lambda x+\frac{\lambda B(\beta+2)}{4} & -\frac{\lambda B \beta}{4} \\
-\frac{\lambda B \beta}{4} & 1-2 \lambda x+\frac{\lambda B(\beta+2)}{4}
\end{array}\right)
$$

hence the eigenvalues are given by

$$
\nu_{\|}=1-2 \lambda x+\frac{\lambda B}{2}, \quad \nu_{\perp}=1-2 \lambda x+\frac{\lambda B}{2}(1+\beta)
$$


It is important to note that the parameter $\beta$ only appears in the transverse eigenvalue $\nu_{\perp}$, so it is a normal parameter, i.e. it has no influence on the dynamics along the invariant submanifold $\Delta$, and only influences the transverse stability: the associated transverse Lyapunov exponent becomes (see the Appendix B)

$$
\Lambda_{\perp}=\lim _{N \rightarrow \infty} \frac{1}{N} \sum_{n=0}^{N} \ln \left|1-2 \lambda x_{n}+\frac{\lambda B}{2}(1+\beta)\right|
$$

This allows us to consider fixed values of the parameters $\lambda$ and $B$, such that a chaotic attractor $A_{s} \subset \Delta$ of the map (17) exists, with an absolutely continuous invariant measure on it. So, we can study the transverse stability of $A_{s}$ as the degree of competition, measured by the parameter $\beta$, varies. Suitable values of the aggregate parameter $\lambda B$, at which chaotic intervals for the restriction (17) exist, are obtained from the well known properties of the logistic map (see e.g. [45], [33]). For example, at the parameter value $\bar{\mu}_{1}=3.5925721841 \ldots$ the period-2 cycle of the logistic map undergoes the homoclinic bifurcation, at which two cyclic chaotic intervals are obtained by the merging of four cyclic chaotic intervals. By using $\lambda B=2\left(\bar{\mu}_{1}-1\right)$ we get a two-band chaotic set $A_{s}$ along the diagonal $\Delta$, and the transverse Lyapunov exponent is shown in Figure 4 as a function of $\beta$.

\section{Fig. 4 APPROX. HERE}

The plot shows the comparison between $\Lambda_{\perp}^{\text {nat }}$, the natural transverse Lyapunov exponent, and $\Lambda_{\perp}^{(2)}$, the transverse Lyapunov exponent associated to the period-2 cycle in $\Delta$. For the values of $\beta$ for which $\Lambda_{\perp}^{\text {nat }}<0$ and

$\Lambda_{\perp}^{(2)}>0$ we can assert that the set $A_{s}$ is certainly a non topological Milnor attractor, since $\Lambda_{\perp}^{\max } \geq \Lambda_{\perp}^{(2)}>0$ (see again the Appendix B). This is the situation shown in Figure 5, obtained for $\beta=0.83$, at which $\Lambda_{\perp}^{\max } \simeq-0.11$ and $\Lambda_{\perp}^{(2)}>0$. The generic trajectory starting from initial conditions taken in the white region of Figure 5 leads to synchronization, whereas the points of the gray region generate interrupted trajectories, involving negative values of the state variables.

\section{Fig. 5 APPROX. HERE}

The Milnor attractor $A_{s}$ is included inside a minimal invariant absorbing area whose boundary can be easily obtained by arcs of critical curves as explained in [13]. This absorbing area, represented in the left panel of Figure 6, constitutes a trapping region inside which the bursts observed during the transient are contained. During the transient, the time evolution of the system is characterized by several bursts away from $\Delta$ before synchronization occurs. The difference $\left\|x_{1}(t)-x_{2}(t)\right\|$ is represented versus time in the right panel of Figure 6 .

\section{Fig. 6 APPROX. HERE}

If the value of $\beta$ is decreased $\Lambda_{\perp}^{\text {nat }}$ goes positive and $A_{s}$ becomes a chaotic saddle i.e. a blowout bifurcation occurs. In case we are studying here the blowout bifurcation is preceded by the contact between critical lines and the basin of negative numbers: the basin of $\Delta$ becomes riddled/intermingled and, after a transient which can also be very long, almost all trajectories goes towards negative values. In the left panel of Figure 7, obtained for $\beta=0.8$, at which $\Lambda_{\perp}^{\text {nat }}>0$ the transient is represented. On the right panel of Figure 7 bursts which characterize the transient are represented as $\left\|x_{1}(t)-x_{2}(t)\right\|$ and exhibit the same time patterns of on-off intermittency.

\section{Fig. 7 APPROX. HERE}

To sum up, when $\Lambda_{\perp}^{\text {nat }}>0$ a small heterogeneity in the initial efforts of identical firms imply that the trajectory obtained never synchronizes, so that the one-dimensional dynamics of the representative agent cannot be used to represent the dynamic evolution of the marketing system.

Another dynamic situation is shown in Figure 8, obtained with parameters $\beta=0.84, B=10$ and $\lambda$ such that $\mu=\bar{\mu}_{1}$, where we can observe the presence of a new attractor $C_{4}$ far from the diagonal $\Delta$. The presence 
of this attractor, whose basin reaches the invariant diagonal $\Delta$ where chaotic dynamics occur, gives rise to the dynamic scenario of global riddling.

\section{Fig. 8 APPROX. HERE}

In fact $C_{4}$ can be reached by a trajectory that starting from an arbitrarily small neighborhood of the transversely unstable 2-cycle embedded in $A_{s}$, namely along a small tongue of the basin of $C_{4}$ located around the transverse unstable manifold issuing from the two periodic points. As in $A_{s}$ there is pure chaos, the preimages of the points of the 2-cycle are densely distributed along $A_{s}$, and the same occurs for the tongues of the basin of the attractor $C_{4}$ located far from $\Delta$. This implies that many trajectories that are locally repelled away from $\Delta$ reach $C_{4}$, whose basin is consequently densely distributed inside the realm of attraction of $A_{s}$, thus giving the typical structure of a riddled basin (see [1]). The two basins of $A_{s} \subset \Delta$ and $C_{4}$ are shown in the Figure 8, however the graphical resolution does not give sufficiently clear idea of such a complexity.

In Figure 9 two trajectories starting from initial conditions very close each other taken in a neighborhood of a periodic point of the transversely unstable cycle of period 2 embedded into $A_{s}$, i.e.

$$
x_{1}^{c}=x_{2}^{c}=\frac{\mu+1 \pm \sqrt{\mu^{2}-2 \mu-3}}{2 \lambda}
$$

are represented, one folded back towards the diagonal $\Delta$ and the other one reaching the attractor $C_{4}$ outside it.

Fig. 9 APPROX. HERE

So, even in this case, two almost identical initial efforts of identical firms give rise to very different long run evolutions, one synchronizing in the long run and the other one converging to a cyclic attractor far from the submanifold where synchronized dynamics occur.

We now consider the model with best reply and inertia (8) with 2 identical firms and $B=1$ :

$$
x_{i}(t+1)=\left(1-\lambda_{i}\right) x_{i}(t)+\lambda_{i}\left(\sqrt{\frac{\sum_{j \neq i} a_{j} x_{j}(t)}{a_{i}}}-\sum_{j \neq i} a_{j} x_{j}(t)\right)
$$

where $\lambda_{i}=\lambda$ and $a_{i}=a, i, j=1,2$. The restriction of (22) to the invariant diagonal is given by

$$
x(t+1)=(1-\lambda) x(t)+\lambda(\sqrt{x(t)}-a x(t))
$$

From the Jacobian matrix in the symmetric case, see (27) in the Appendix A, the normal and transversal Lyapunov exponent are obtained, respectively given by

$$
\begin{aligned}
& \Lambda_{\|}=\lim _{n \rightarrow \infty} \frac{1}{n} \sum_{k=0}^{n} \ln \left|1-\lambda+\lambda\left(\frac{1}{2} \sqrt{\frac{1}{x_{k}}}-a\right)\right| \\
& \Lambda_{\perp}=\lim _{n \rightarrow \infty} \frac{1}{n} \sum_{k=0}^{n} \ln \left|1-\lambda-\lambda\left(\frac{1}{2} \sqrt{\frac{1}{x_{k}}}-a\right)\right|
\end{aligned}
$$

Both $\Lambda_{\|}$and $\Lambda_{\perp}$ depend both on $a$ and $\lambda$, so there are no normal parameters. We notice that for $\lambda=1$ (no inertia) we have $\Lambda_{\perp}=\Lambda_{\|}$and they are showed in Figure 10 as the parameter $a$ varies.

Fig. 10 APPROX. HERE

In this case every stable cycle of period $n, C^{n}$, is a topological attractor characterized both by negative Lyapunov exponents. Pure chaos exists in a set of positive Lebesgue measure in the parameter space (see [28], or [49] for a survey) and, since $\Lambda_{\perp}^{\text {nat }}>0$, subsets $A_{s}^{k} \in \Delta$ in which chaotic dynamics occur are chaotic saddles. 
As stressed in [22], synchronization can occur for certain parameters' constellations giving negative values of $\Lambda_{\perp}$ (and for certain initial conditions) as showed in Figure 11. However, in the same Figure we observe other attractors in the two-dimensional phase space out of $\Delta$ coexisting with the stable cycles on the diagonal. In other words, even if stable cycles may exist embedded into the diagonal $\Delta$, their basins may be quite intermingled with the basins of other stable cycles located outside $\Delta$.

\section{Fig. 11 APPROX. HERE}

When no stable cycles exist along $\Delta$, than trajectories never synchronize, except the non generic case of identical firms that start with identical initial conditions, i.e. only for a set of initial conditions belonging to $\Delta$ which has zero Lebesgue measure in $\mathbb{R}^{2}$. It is plain that in this case the one-dimensional synchronized dynamics of the representative agent has no economic meaning. In Figure 12 an example of a typical dynamic scenario of the model (22) with $\Lambda_{\perp}^{\text {nat }}>0$ is shown.

Fig. 12 APPROX. HERE

\subsection{Quasi-homogeneous firms and symmetry loss}

In the previous subsection we made the very restrictive assumption that the firms' structural parameters are the same and the difference between the competitors lies only in their initial effort allocations. Although synchronization does not necessarily occur for all initial effort allocations, we could determine for which parameters' constellations and initial conditions the two-dimensional model may be substituted by the one-dimensional model of a representative agent as far as long run dynamics is concerned.

For practical purposes, we now check, by using the marketing model (15) as a paradigmatic example, what happens as a consequence of small heterogeneities due to small parameters' mismatches. We notice, first of all, that a parameters' mismatch causes the destruction of the invariance of $\Delta$, due to the fact that the map is no longer symmetric. This may lead to quite different dynamics, since after the parameters' mismatch synchronization along $\Delta$ can no longer occur, and the generic trajectory fills up the minimal absorbing area around the former invariant set $A_{s}$ (see e.g. [8], [13]). However, if the attractor $A_{s}$ existing along $\Delta$ before the parameters' mismatch is a topological attractor, that is $\Lambda_{\perp}^{\max }<0$, then the introduction of small heterogeneities does not have such a disruptive effect, and the symmetric model still serves as a good approximation of the behavior of the two firms. Such a situation is shown in the Figure 13, where we started from a situation in which identical firms are characterized by a set of parameters such that the one-dimensional chaotic attractor embedded into the diagonal is an asymptotic attractor, i.e. $\Lambda_{\perp}^{\max }<0$. We remark that this is not easy to be proved in general, since the cycles included in a chaotic attractor are infinitely many. However, we claim the fact that the natural transverse Lyapunov exponent has a strong negative value, as shown in Figure 4, and the periodic cycles of lower period are transversely stable. This last point constitutes a well known conjecture, based on the fact that if $\Lambda_{\perp}^{\text {nat }}<0$ and $\Lambda_{\perp}^{\max }>0$, then some low period cycles should be transversely unstable, because if a cycle of high period is transversely unstable, i.e. its transverse Lyapunov exponent is positive, also $\Lambda_{\perp}^{\text {nat }}$ should be positive, see e.g. [34].

\section{Fig. 13 APPROX. HERE}

So, the fact that the chaotic set on which synchronized dynamics occur is an asymptotic attractor for the two-dimensional map implies two things: first, the synchronization of trajectories starting out of it, and in its basin, is very fast; second, if we introduce a small parameter mismatch, the resulting trajectories are "almost synchronized" (see Figure 13). Instead, if the introduction of a parameters' mismatch occurs starting from a symmetric situation where the chaotic one-dimensional invariant set $A_{s} \subset \Delta$ is an attractor only in Milnor sense (i.e. not asymptotic) then a quite different effect generally occurs, because after the symmetry breaking 
endless intermittency filling up the minimal absorbing area around $A_{s}$ is observed. This is illustrated in Figure 14, obtained starting from the situation already discussed in the previous subsection and illustrated in Figure 6 after the introduction of a very small difference between the response parameters of the firms, namely $a_{1}=a_{2}$ and $\beta_{1}=0.83$ while $\beta_{2}=\beta_{1}-10^{-5}$. Such a small perturbation leads to quite different dynamics, since synchronization no longer occurs, and the bursts never stop. The generic trajectory fills up the absorbing area, which now appears to be a two-dimensional chaotic area, as shown in Figure 14. In the left panel we show the evolution of the system starting from the initial effort allocation $\left(x_{1}(0), x_{2}(0)\right)=(3.5,3.5) \in \Delta$, i.e., from homogeneous initial choices, is represented in the phase space $\left(x_{1}, x_{2}\right)$, whereas in the right Figure the difference of the marketing efforts over time, $\left(x_{1}(t)-x_{2}(t)\right)$, is represented over 5000 periods. It is evident that long time intervals exist in which the two firms show quasi-synchronized behavior, but in-between such intervals asynchronous behavior emerges with an apparently random pattern.

Fig. 14 APPROX. HERE

Of course, the study of the effects of small parameters' mismatches may be important in economic dynamic modelling, as stressed in [9] and [31].

\section{Conclusions}

In this paper we have critically considered a common practice, in economic literature, that consists in the reduction of a model describing $n$ identical agents to a one-dimensional dynamical system that governs the asymptotic dynamics of a representative agent that summarizes the common behaviour of the $n$ identical firms. Often the same equation is assumed to represent the dynamics of the system even in the presence of "sufficiently small" heterogeneities, and is often used to study the trade-off between stability of the overall system and the number $n$ of firms, that can be seen as a bifurcation parameter of the one-dimensional restriction. This approach can be found in several books and papers, see e.g. [50], [48], [22], [14].

In this paper, starting from some market share attraction models commonly used in the literature to describe marketing dynamics with adaptive firms, we have shown how dangerous (sometimes even misleading) this approach may be. First of all, we have shown that similar nonlinear models based on adaptive profit increasing mechanism, that exhibit similar and quite rich dynamic behaviours, may collapse into qualitatively different one-dimensional models (and sometimes even leading to opposite stability statements) when identical agents are considered. Moreover, by using some advanced tools from the recent literature on symmetric dynamic models, we have shown under which conditions the one-dimensional restriction is robust with respect to the introduction of small asymmetries in the initial conditions and/or in the parameters of the model. The theoretical as well as the numerical results suggest that the study of the transverse Lyapunov exponents gives important suggestions about both questions. In fact, negative values of the transverse Lyapunov exponent guarantees synchronization in the long run (sometimes after a long transient characterized by on-off intermittency) so that the one-dimensional reduction of the system remains meaningful even if the identical firms start from (slightly) different initial conditions. However the question of coexistence of attracting sets, with the related phenomenon of riddled basins, should be carefully considered as well. Analogously, when small parameters' mismatches are introduced, if all the transverse Lyapunov exponents are negative then only small changes in the shape of the attractor are observed, so that the long run dynamics of quasi-identical agents are still well approximated by the one-dimensional model. Instead, in the presence of some positive transverse Lyapunov exponents (even if the natural transverse Lyapunov exponent is negative, so that the one-dimensional invariant set along the diagonal is an attractor only in Milnor sense) a quite different asymptotic dynamics may arise, along an attractor whose shape and extension depend on the global properties of the dynamical system.

These results suggest that even if the restriction of the complete dynamical system to the diagonal (where synchronized dynamics occur) may be meaningful in some cases, in general this is not the case (see also [31] on this point). 
In order to address this general idea in the simplest case, the examples and numerical simulations given in this paper are referred to marketing models with just two firms, represented by two-dimensional dynamical systems. Similar arguments may be applied to higher dimensional models, that represent systems with more than two firms, where conditions for complete synchronization (i.e. all firms synchronize) or partial synchronization (i.e. only a subset of firms synchronize) may be given. This will be a natural continuation of the research stream whose early modest steps are moved by this paper.

From an economic point of view, the results of this paper just make us aware how an assumptions made in (or almost throughout) the economic literature should be critically considered. In fact, if the assumption of aggregate dynamics where all identical agents are summarized by the behaviour of a representative agent is made for analytical tractability, we should be aware that such aggregation represents a very special case. The reason is that for dynamic models the symmetric case is often non-generic.

\section{Acknowledgements.}

This work has been performed within the activity of the PRIN project "Local interactions and global dynamics in economics and finance: models and tools", MIUR, Italy and in the framework of COST action IS1104, "The EU in the new complex geography of economic systems: models, tools and policy evaluation". The authors thank Laura Gardini, Michael Kopel and an anonymous referee for their useful comments. The usual disclaimers apply.

\section{Appendix A. Study of the stability of the steady states of the three models of section 2 with two firms}

For simpler notations we set:

$$
x_{i}^{\prime}:=x_{i}(t+1) ; x_{i}:=x_{i}(t)
$$

\subsection{Model (8) with $n=2$}

Let us consider the model (8) with $n=2$, given by

$$
T:\left\{\begin{array}{l}
x_{1}^{\prime}=\left(1-\lambda_{1}\right) x_{1}+\lambda_{1}\left(\sqrt{B \frac{a_{2} x_{2}}{a_{1}}}-a_{2} x_{2}\right) \\
x_{2}^{\prime}=\left(1-\lambda_{2}\right) x_{2}+\lambda_{2}\left(\sqrt{B \frac{a_{1} x_{1}}{a_{2}}}-a_{1} x_{1}\right)
\end{array}\right.
$$

The fixed points are the solutions of the algebraic system

$$
T:\left\{\begin{aligned}
x_{1} & =\sqrt{B \frac{a_{2} x_{2}}{a_{1}}}-a_{2} x_{2} \\
x_{2} & =\sqrt{B \frac{a_{1} x_{1}}{a_{2}}}-a_{1} x_{1}
\end{aligned}\right.
$$

This system can be analytically solved in the symmetric case $a_{1}=a_{2}=a$, and the following fixed points are obtained: $O=(0,0) ; E^{*}=\frac{B}{(1+a)^{2}}(1,1)$, both located along the invariant diagonal $\Delta$, and, for $a \geq 3$, two further fixed points in symmetric positions with respect to $\Delta$

$$
\begin{aligned}
& E_{1}^{*}=\frac{B}{(a-1)^{2}(a+1)}(a-1+\sqrt{(a+1)(a-3)}, a-1-\sqrt{(a+1)(a-3)}) \\
& E_{2}^{*}=\frac{B}{(a-1)^{2}(a+1)}(a-1-\sqrt{(a+1)(a-3)}, a-1+\sqrt{(a+1)(a-3)})
\end{aligned}
$$


The conditions for the stability of the central positive equilibrium $E^{*}$ are obtained from the Jacobian matrix

$$
\boldsymbol{J}\left(x_{1}, x_{2}\right)=\left(\begin{array}{cc}
1-\lambda_{1} & \lambda_{1}\left(\frac{1}{2} \sqrt{\frac{B}{x_{2}}}-a\right) \\
\lambda_{2}\left(\frac{1}{2} \sqrt{\frac{B}{x_{1}}}-a\right) & 1-\lambda_{2}
\end{array}\right)
$$

computed at the fixed point

$$
\boldsymbol{J}\left(E^{*}\right)=\left(\begin{array}{cc}
1-\lambda_{1} & \lambda_{1} \frac{1-a}{2} \\
\lambda_{2} \frac{1-a}{2} & 1-\lambda_{2}
\end{array}\right)
$$

In fact, from the characteristic equation

$$
P(z)=z^{2}-T r \cdot z+D e t=0,
$$

where $\operatorname{Tr}=2-\lambda_{1}-\lambda_{2}$ and $\operatorname{Det}=\left(1-\lambda_{1}\right)\left(1-\lambda_{2}\right)-\lambda_{1} \lambda_{2}(1-a)^{2} / 4$ are the trace and the determinant of $\boldsymbol{J}\left(E^{*}\right)$ respectively, a sufficient condition for the stability is expressed by the following system of inequalities

$$
\begin{array}{r}
P(1)=1-T r+\text { Det }>0 \\
P(-1)=1+T r+\text { Det }>0 \\
1-\text { Det }>0
\end{array}
$$

that give necessary and sufficient conditions for the two eigenvalues be inside the unit circle of the complex plane. In our case

$$
\begin{aligned}
P(1) & =\lambda_{1} \lambda_{2}\left(1-\frac{(1-a)^{2}}{4}\right)>0 \Longrightarrow a<3 \\
P(-1) & >0 \Longrightarrow a<a_{f} \\
1-\text { Det } & =\lambda_{1}+\lambda_{2}-\lambda_{1} \lambda_{2}+\lambda_{1} \lambda_{2} \frac{(1-a)^{2}}{4}>0 \Longrightarrow \forall a, \lambda_{i} \in[0,1]
\end{aligned}
$$

where

$$
a_{f}:=1+2 \sqrt{1+2 \frac{2-\lambda_{1}-\lambda_{2}}{\lambda_{1} \lambda_{2}}} .
$$

Hence, the equilibrium is stable for $a<3$. At the bifurcation value $a=3$ a pitchfork bifurcation occurs at which $E^{*}$ becomes a saddle point and the two equilibriums $E_{1}^{*}$ and $E_{2}^{*}$ are created. Moreover, at $a=a_{f} \geq 3$ a flip bifurcation of $E^{*}$ occurs at which $E^{*}$ is transformed into an unstable node and a saddle cycle of period 2 is created. Notice that for $\lambda_{1}=\lambda_{2}=1$ (the case of best reply without inertia) $a_{f}=3$, so the pitchfork and flip bifurcations occur simultaneously.

\subsection{Model (10) with $n=2$}

Let us consider the model (15) for $n=2$, given by

$$
T:\left\{\begin{array}{l}
x_{1}^{\prime}=x_{1}+\lambda_{1} x_{1}\left(B \frac{a_{1} x_{1}^{\beta_{1}}}{a_{1} x_{1}^{\beta_{1}}+a_{2} x_{2}^{\beta_{2}}}-x_{1}\right) \\
x_{2}^{\prime}=x_{2}+\lambda_{2} x_{2}\left(B \frac{a_{2} x_{2}^{\beta_{2}}}{a_{1} x_{1}^{\beta_{1}}+a_{2} x_{2}^{\beta_{2}}}-x_{2}\right)
\end{array}\right.
$$


Its fixed points are the solutions of the system

$$
\left\{\begin{array}{l}
x_{1}\left(B \frac{a_{1} x_{1}^{\beta_{1}}}{a_{1} x_{1}^{\beta_{1}}+a_{2} x_{2}^{\beta_{2}}}-x_{1}\right)=0 \\
x_{2}\left(B \frac{a_{2} x_{2}^{\beta_{2}}}{a_{1} x_{1}^{\beta_{1}}+a_{2} x_{2}^{\beta_{2}}}-x_{2}\right)=0
\end{array}\right.
$$

There are three evident "boundary solutions":

$$
O=(0,0) ; E_{1}=(B, 0) ; E_{2}=(0, B)
$$

but $O$ is not a fixed point because the map is not defined in it. There is also a positive fixed point, given by the solution of the system

$$
\left\{\begin{array}{l}
B \frac{a_{1} x_{1}^{\beta_{1}}}{a_{1} x_{1}^{\beta_{1}}+a_{2} x_{2}^{\beta_{2}}}-x_{1}=0 \\
B \frac{a_{2} x_{2}^{\beta_{2}}}{a_{1} x_{1}^{\beta_{1}}+a_{2} x_{2}^{\beta_{2}}}-x_{2}=0
\end{array}\right.
$$

It is possible to see that one and only one solution exists given by

$$
E^{*}=\left(x^{*}, B-x^{*}\right)
$$

with $x^{*} \in(0, B)$ unique solution of the equation

$$
F(x)=\left(\frac{a_{2}}{a_{1}}\right)^{1 /\left(1-\beta_{2}\right)} x^{\left(1-\beta_{1}\right) /\left(1-\beta_{2}\right)}+x-B=0
$$

obtained from (32) after some algebraic manipulations. In fact, $F$ is a continuous function with $F(0)<0$, $F(B)>0$ and $F^{\prime}(x)>0$ for each $x>0$. An analytic expression of the solution is obtained in the case $\beta_{1}=\beta_{2}=\beta$, given by

$$
x^{*}=\frac{B}{1+\left(\frac{a_{2}}{a_{1}}\right)^{\frac{1}{(1-\beta)}}}
$$

Moreover, under the further assumption $a_{2} / a_{1}=1$, i.e. in the case of identical firms, we get

$$
E^{*}=\left(\frac{B}{2}, \frac{B}{2}\right)
$$

With a given set of parameters $B, \beta_{1}$ and $\beta_{2}$ the positive fixed point $E^{*}$ is locally asymptotically stable for sufficiently small values of the adjustment speeds $\lambda_{1}$ and $\lambda_{2}$ and, as usual in dynamic models with adaptive adjustment, the fixed point $E^{*}$ loses stability as one or both of the adjustment speeds are increased, after which more complex attractors are created around the unstable fixed point (see [7], where these results are obtained through a standard study of the local stability of the positive fixed point, obtained by a numerical solution of the characteristic equation for the localization, in the complex plane, of the eigenvalues of the Jacobian matrix). In this paper we are mainly interested in the symmetric case of identical firms, for which the Jacobian matrix (19) computed at $E^{*}$ becomes

$$
\boldsymbol{J}\left(E^{*}\right)=\left(\begin{array}{cc}
1-\frac{\lambda B}{2}(1-\beta / 2) & -\frac{\lambda B \beta}{4} \\
-\frac{\lambda B \beta}{4} & 1-\frac{\lambda B}{2}(1-\beta / 2)
\end{array}\right)
$$

hence the eigenvalues at the positive fixed point are $\lambda_{\|}=1-\frac{1}{2} \lambda B$, with eigendirection along $\Delta$ and $\lambda_{\perp}=$ $1-\frac{1}{2} \lambda B(1-\beta)$ with eigendirection orthogonal to $\Delta$. It is easy to see that the steady state $E^{*}$ is locally asymptotically stable for $\lambda B<4$ and $0<\lambda B(1-\beta)<4$, however only the first condition is important as only values of $\beta_{i} \in(0,1]$ are meaningful in applications, see [20]. 


\subsection{Model (13) with $n=2$}

For the model (13) with $n=2$, given by

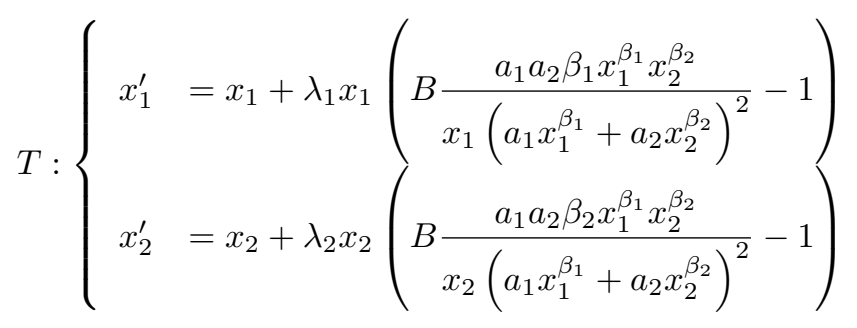

The fixed points are the solutions of the system

$$
\left\{\begin{array}{l}
a_{1} a_{2} B \beta_{1} x_{1}^{\beta_{1}} x_{2}^{\beta_{2}}-x_{1}\left(a_{1} x_{1}^{\beta_{1}}+a_{2} x_{2}^{\beta_{2}}\right)^{2}=0 \\
a_{1} a_{2} B \beta_{2} x_{1}^{\beta_{1}} x_{2}^{\beta_{2}}-x_{2}\left(a_{1} x_{1}^{\beta_{1}}+a_{2} x_{2}^{\beta_{2}}\right)^{2}=0
\end{array}\right.
$$

The solutions must belong to the line

$$
x_{2}=\frac{\beta_{2}}{\beta_{1}} x_{1}
$$

and plugging this equation into the first equilibrium condition we obtain $x_{1}^{\beta_{1}+\beta_{2}} F\left(x_{1}\right)=0$, where

$$
F(x)=\frac{a_{2}}{a_{1}}\left(\frac{\beta_{2}}{\beta_{1}}\right)^{\beta_{2}}\left(B \beta_{1}-2 x\right)-x^{\beta_{1}-\beta_{2}+1}-\left(\frac{a_{2}}{a_{2}}\right)^{2}\left(\frac{\beta_{2}}{\beta_{1}}\right)^{2 \beta_{2}} x^{\beta_{2}-\beta_{1}+1}
$$

If $x^{*}>0$ is a zero of the function $F$, then the point $E^{*}=\left(x_{1}^{*}, x_{2}^{*}\right)$, where $x_{2}^{*}$ is computed according to $(39)$, is a fixed point of (37). As $F(x)$ is continuous with

$$
\begin{gathered}
F(0)=\frac{a_{2}}{a_{1}}\left(\frac{\beta_{2}}{\beta_{1}}\right)^{\beta_{2}} B \beta_{1}>0 \\
F\left(\frac{B \beta_{1}}{2}\right)=-\left[x^{\beta_{1}-\beta_{2}+1}+\left(\frac{a_{2}}{a_{1}}\right)^{2}\left(\frac{\beta_{2}}{\beta_{1}}\right)^{2 \beta_{2}} x^{\beta_{2}-\beta_{1}+1}\right]<0
\end{gathered}
$$

then a solution $x^{*} \in\left(0, \frac{B \beta_{1}}{2}\right)$ exists. Moreover, as

$$
F^{\prime}(x)=-2 \frac{a_{2}}{a_{1}}\left(\frac{\beta_{2}}{\beta_{1}}\right)^{\beta_{2}}+\left(\beta_{1}-\beta_{2}+1\right) x^{\beta_{1}-\beta_{2}}+\left(\frac{a_{2}}{a_{1}}\right)^{2}\left(\frac{\beta_{2}}{\beta_{1}}\right)^{2 \beta_{2}}\left(\beta_{2}-\beta_{1}+1\right) x^{\beta_{2}-\beta_{1}}
$$

uniqueness of such solution is ensured for $x>0$ provided that $\beta_{1}-\beta_{2}+1 \geq 0$ and $\beta_{2}-\beta_{1}+1 \geq 0$, a condition usually satisfied in applications, due to the conditions $\beta_{i} \in(0,1]$ (see [20]). Moreover it is always trivially satisfied in the case of equal elasticities $\beta_{1}=\beta_{2}$. Indeed, in the case of identical firms $\beta_{1}=\beta_{2}=\beta$; $a_{1}=a_{2}$, the function $F(x)$ becomes $F(x)=B \beta-4 x$, hence the unique equilibrium is

$$
E^{*}=\left(x^{*}, x^{*}\right) \text { with } x^{*}=\frac{B \beta}{4}
$$


The Jacobian matrix $\boldsymbol{J}$ has entries

$$
\begin{aligned}
& J_{11}=1-\lambda_{1}-\lambda_{1} B a_{1} a_{2} \beta_{1}^{2} x_{1}^{\beta_{1}-1} x_{2}^{\beta_{2}} \frac{a_{1} x_{1}^{\beta_{1}}-a_{2} x_{2}^{\beta_{2}}}{\left(a_{1} x_{1}^{\beta_{1}}+a_{2} x_{2}^{\beta_{2}}\right)^{3}} \\
& J_{12}=\lambda_{1} \beta_{1} \beta_{2} B a_{1} a_{2} x_{1}^{\beta_{1}} x_{2}^{\beta_{2}-1} \frac{a_{1} x_{1}^{\beta_{1}}-a_{2} x_{2}^{\beta_{2}}}{\left(a_{1} x_{1}^{\beta_{1}}+a_{2} x_{2}^{\beta_{2}}\right)^{3}} \\
& J_{21}=\lambda_{2} \beta_{1} \beta_{2} B a_{1} a_{2} x_{1}^{\beta_{1}-1} x_{2}^{\beta_{2}} \frac{a_{2} x_{2}^{\beta_{2}}-a_{1} x_{1}^{\beta_{1}}}{\left(a_{1} x_{1}^{\beta_{1}}+a_{2} x_{2}^{\beta_{2}}\right)^{3}} \\
& J_{22}=1-\lambda_{2}-\lambda_{2} B a_{1} a_{2} \beta_{2}^{2} x_{1}^{\beta_{1}} x_{2}^{\beta_{2}-1} \frac{a_{2} x_{2}^{\beta_{2}}-a_{1} x_{1}^{\beta_{1}}}{\left(a_{1} x_{1}^{\beta_{1}}+a_{2} x_{2}^{\beta_{2}}\right)^{3}}
\end{aligned}
$$

that computed at a point of the diagonal $\Delta$ (and, in particular, at the equilibrium $E^{*}$ ) becomes a multiple of the identity matrix

$$
\boldsymbol{J}(x, x)=(1-\lambda) \mathbf{I}
$$

so that the equilibrium is an attracting star node or a repelling star node according to $\lambda<2$ or $\lambda>2$ respectively. At $\lambda=2$ a degenerate flip bifurcation occurs.

\section{Appendix B. Chaos synchronization, transverse stability, Milnor attractors and related bifurcations in two-dimensional models.}

Let us consider a dynamic model represented by a map of the plane into itself $T:\left(x_{1}, x_{2}\right) \rightarrow\left(x_{1}^{\prime}, x_{2}^{\prime}\right)$. Let us assume, like in the case of identical competitors, that the map remains the same if the variables $x_{1}$ and $x_{2}$ are swapped, i.e. $T \circ S=S \circ T$, where $S:\left(x_{1}, x_{2}\right) \rightarrow\left(x_{2}, x_{1}\right)$ is the reflection through the diagonal

$$
\Delta=\left\{\left(x_{1}, x_{2}\right) \in \mathbb{R}^{2} \mid x_{1}=x_{2}\right\} .
$$

This symmetry property implies that the diagonal is mapped into itself, i.e., $T(\Delta) \subseteq \Delta$, which corresponds with the obvious statement that, in a deterministic framework, identical competitors, starting from identical initial conditions, behave identically for each time. The trajectories embedded into $\Delta$, i.e. characterized by $x_{1}(t)=x_{2}(t)$ for every $t$, are called synchronized trajectories, and they are governed by the one-dimensional map given by the restriction of $T$ to the invariant submanifold $\Delta$

$$
x(t+1)=f(x(t))=T_{\Delta}(x(t)) \text { with } T_{\Delta}=\left.T\right|_{\Delta}: \Delta \rightarrow \Delta .
$$

A trajectory starting out of $\Delta$, i.e. with $x_{1}(0) \neq x_{2}(0)$, is said to synchronize if $\left\|x_{1}(t)-x_{2}(t)\right\| \rightarrow 0$ as $t \rightarrow+\infty$. A question which naturally arises is whether identical competitors starting from different initial conditions will synchronize in the long run, so that the asymptotic behavior is governed by the simpler one-dimensional model (43). This question can be reformulated as follows. Let $A_{s}$ be an attractor of the one-dimensional map (43). Is it also an attractor for the two-dimensional map $T$ ?

To answer this question let us consider the Jacobian matrix of $T$ computed at any point of $\Delta$, say $\boldsymbol{J}=$ $\left\{J_{i j}(x)\right\}$ with the double symmetry property $J_{11}=J_{22}$ and $J_{12}=J_{21}$. The two orthogonal eigenvectors of such a symmetric matrix are one parallel to $\Delta$, say $\mathbf{v}_{\|}=(1,1)$, and one perpendicular to it, say $\mathbf{v}_{\perp}=(1,-1)$, with related eigenvalues given by

$$
\lambda_{\|}(x)=J_{11}(x)+J_{12}(x) \quad \text { and } \quad \lambda_{\perp}(x)=J_{11}(x)-J_{12}(x)
$$


Of course, $\lambda_{\|}(x)=f^{\prime}(x)$. Since the product of matrices with the structure of $J$ has the same structure as well, a $k$-cycle $\left\{s_{1}, \ldots, s_{k}\right\}$ embedded into $\Delta$ has eigenvalues $\lambda_{\|}^{k}=\prod_{i=1}^{k} \lambda_{\|}\left(s_{i}\right)$ and $\lambda_{\perp}^{k}=\prod_{i=1}^{k} \lambda_{\perp}\left(s_{i}\right)$, with eigenvectors $\mathbf{v}_{\|}$and $\mathbf{v}_{\perp}$ respectively. So, an answer to the question stated above requires a study of the transverse stability, i.e. stability in the direction orthogonal to $\Delta$. If $A_{s}$ is a cycle, then the study of the transverse stability is the usual one, based on the modulus of the eigenvalues of the cycle in the direction transverse to $\Delta$. The problem becomes more interesting when $A_{s}$ is a chaotic attractor. Indeed, dynamical systems with chaotic trajectories embedded into an invariant submanifold of lower dimensionality than the total phase space have raised an increasing interest in the scientific community because the phenomenon of chaos synchronization may occur (see e.g. [25], [41], [27], [32]) i.e., the time evolution of the two competitors synchronize in the long run even if each of them behaves chaotically. The key property for the study of the transverse stability of a chaotic attractor $A_{s} \subset \Delta$ is that it includes infinitely many periodic orbits which are unstable in the direction along $\Delta$. In this case, Milnor attractors (see [36]) which are not stable in Lyapunov sense appear quite naturally in this context. To better understand the meaning of this point, we recall some definitions.

Let $\mathcal{A}$ be a closed invariant set such that $T(\mathcal{A}) \equiv \mathcal{A}$, and let $\mathcal{B}(\mathcal{A})$ denote its basin of attraction, i.e. is the set of points whose $\omega$-limit set belongs to $\mathcal{A}$.

Definition. $\mathcal{A}$ is an asymptotically stable attractor (or topological attractor) if it is Lyapunov stable, i.e. for every neighborhood $U$ of $\mathcal{A}$ there exists a neighborhood $V$ of $\mathcal{A}$ such that $T^{t}(V) \subset U \forall t \geq 0$, and $B(\mathcal{A})$ contains a neighborhood of $\mathcal{A}$.

In other words, if $\mathcal{A}$ is a topological attractor then a neighborhood $W \supset \mathcal{A}$ exists such that $T^{t}(\mathbf{x}) \rightarrow \mathcal{A}$ as $t \rightarrow+\infty$ for any $\mathbf{x} \in W$. In this case the basin $\mathcal{B}(\mathcal{A})$ is an open set given by $\mathcal{B}(\mathcal{A})=\bigcup_{t \geq 0} T^{-t}(W)$.

Definition. A closed invariant set $\mathcal{A}$ is said to be a weak attractor in Milnor sense (or simply Milnor attractor) if its basin of attraction $B(\mathcal{A})$ has positive Lebesgue measure.

Note that a topological attractor is also a Milnor attractor, whereas the converse is not true. The more general notion of Milnor attractor has been introduced to evidence the existence of invariant sets which "attract" many points even if they are not attractors in the usual topological sense. In this case, [36] denotes $\mathcal{B}(\mathcal{A})$ as "Realm of attraction", reserving the term "basin" when $\mathcal{B}(\mathcal{A})$ is an open set. However, since the term basin is more standard in the literature, we shall use such term even when $\mathcal{A}$ is a Milnor (but not topological) attractor, for which $\mathcal{B}(\mathcal{A})$ is not, in general, an open set.

We now recall some definitions and results related to the problem of chaos synchronization, see e.g. [17], [4]. Let $A_{s}$ be a chaotic attractor (with absolutely continuous invariant measure on it) of the restriction (43) of $T$ to $\Delta$. Its attractivity in the two-dimensional phase space is given in terms of the transverse Lyapunov exponents

$$
\Lambda_{\perp}=\lim _{N \rightarrow \infty} \frac{1}{N} \sum_{i=0}^{N} \ln \left|\lambda_{\perp}\left(s_{i}\right)\right|
$$

where $\left\{s_{i}=f^{i}\left(s_{0}\right), i \geq 0\right\}$ is a trajectory embedded in $A_{s}$. For a chaotic set $A_{s} \subset \Delta$, infinitely many transverse Lyapunov exponents can be defined: If $x(0)$ belongs to a $k$-cycle then $\Lambda_{\perp}=\ln \left|\lambda_{\perp}^{k}\right|$, so that the cycle is transversely stable if $\Lambda_{\perp}<0$, whereas if $x(0)$ belongs to a generic aperiodic trajectory embedded inside the chaotic set $A_{s}$ then $\Lambda_{\perp}$ is the natural transverse Lyapunov exponent $\Lambda_{\perp}^{\text {nat }}$, where the term "natural" means that the Lyapunov exponent associated to the natural, or SBR (Sinai-Bowen-Ruelle), measure, i.e., computed for a typical trajectory taken in the chaotic attractor $A_{s} . \Lambda_{\perp}^{\text {nat }}$ gives the "average" local behavior of the trajectories in a neighborhood of the invariant set $A_{s}$ and allows one to detect new kinds of bifurcations such as the riddling bifurcation or the blowout bifurcation. Since infinitely many cycles, all unstable along $\Delta$, are embedded inside a chaotic attractor $A_{s}$, a spectrum of transverse Lyapunov exponents can be defined, see e.g. [17]

$$
\Lambda_{\perp}^{\min } \leq \ldots \leq \Lambda_{\perp}^{\text {nat }} \leq \ldots \leq \Lambda_{\perp}^{\max }
$$

The meaning of the inequalities in (45) can be intuitively understood on the basis of the property that $\Lambda_{\perp}^{\text {nat }}$ expresses a sort of "weighted balance" between the transversely repelling and transversely attracting cycles (see 
e.g. [38]). If $\Lambda_{\perp}^{\max }<0$, i.e. all the cycles embedded in $A_{s}$ are transversely stable, then $A_{s}$ is asymptotically stable, in the usual Lyapunov sense, for the two-dimensional map $T$. However, it may occur that some cycles embedded in the chaotic set $A_{s}$ become transversely unstable, i.e. $\Lambda_{\perp}^{\max }>0$, while $\Lambda_{\perp}^{\text {nat }}<0$. In this case, $A_{s}$ is no longer Lyapunov stable, but it continues to be a Milnor attractor, i.e. it attracts a positive (Lebesgue) measure set of points of the two-dimensional phase space. So, if $\mathcal{A} \subset \Delta$ is a chaotic attractor of $\left.T\right|_{\Delta}$ with absolutely continuous invariant measure, then a sufficient condition for a $\mathcal{A}$ be a Milnor, but not topological, attractor for the two-dimensional map $T$, is that: (i) at least one $k$-cycle embedded in $\mathcal{A}$ is transversely repelling, i.e. $\Lambda_{\perp}^{\max }>0$, and (ii) the Lyapunov exponent $\Lambda_{\perp}^{\text {nat }}$ is negative. This means that the majority of the trajectories on $\mathcal{A}$ are transversely attracting, but some (even infinitely many) trajectories inside $\mathcal{A}$ can exist whose transverse Lyapunov exponent is positive. In other words, transversely repelling trajectories can be embedded into a chaotic set which is attracting only "on average".

The transition from asymptotic stability to attractivity only in Milnor sense, marked by a change of sign of $\Lambda_{\perp}^{\max }$ from negative to positive, is denoted as the riddling bifurcation (or bubbling bifurcation). Even if the occurrence of such bifurcations is detected through the study of the transverse Lyapunov exponents, their effects depend on the action of the nonlinearities far from $\Delta$, that is, on the global properties of the dynamical system. In fact, after the riddling bifurcation two possible scenarios can be observed according to the fate of the trajectories that are locally repelled along (or near) the local unstable manifolds of the transversely repelling cycles (see e.g. [39], [1], [34], [13]):

(L) they can be reinjected towards $\Delta$, so that the dynamics of such trajectories are characterized by some bursts far from $\Delta$ before synchronizing on it (a very long sequence of such bursts, which can be observed when $\Lambda_{\perp}$ is close to zero, has been called on-off intermittency, see e.g. [52], [4]);

$(\mathbf{G})$ they may belong to the basin of another attractor, in which case the phenomenon of riddled basins is obtained, see [1], [39].

Some authors call local riddling the situation (L) and, by contrast, global riddling the situation (G) (see [4], [33]). As shown in [13], see also [8], the reinjection of the locally repelled trajectories can be usefully described by the method of critical curves and their folding action (see [37] for more details on critical curves). When also $\Lambda_{\perp}^{\text {nat }}$ becomes positive, due to the fact that the transversely unstable periodic orbits embedded into $A_{s}$ have a greater weight as compared with the stable ones, a blowout bifurcation occurs, after which $A_{s}$ is no longer a Milnor attractor, because it attracts a set of points of zero measure, and becomes a chaotic saddle, see [17]. Also the macroscopic effect of a blowout bifurcation is strongly influenced by the behavior of the dynamical system far from the invariant submanifold $\Delta$ : The trajectories starting close to the chaotic saddle may be attracted by some attracting set far from $\Delta$ or remain inside a two-dimensional compact set located around the chaotic saddle $A_{s}$, inside which on-off intermittency occurs.

As noticed by many authors, (see e.g. [4], [17], [27], [34], [12], [13]), even if the occurrence of riddling and blowout bifurcations is detected through the transverse Lyapunov exponents, i.e. from a local analysis of the linear approximation of the map along $\Delta$, their effects are determined by the global properties of the map.

\section{References}

[1] Alexander J.C. , J.A. Yorke, Z. You and I. Kan, Riddled basins, Int. Jou. of Bif. \& Chaos, 2 (1992) 795-813

[2] Aoki M. , New Approaches to Macroeconomic Modelling, Cambridge University Press, New York, 1996.

[3] Arrow K.J. and Hurwicz L. "Stability of the gradient process in n-persons games" Journal of the Society for Industrial and Applied Mathematics, 8 (2) (1960) 280-294

[4] Ashwin P., J. Buescu and I. Stewart, From attractor to chaotic saddle: a tale of transverse instability, Nonlinearity, 9 (1996) 703-737 
[5] Baumol W.J. and R.E. Quandt, "Rules of Thumb and optimally imperfect decisions", The American Economic Review, 54 (1964) 23-46.

[6] Bell D.E., R.L. Keeney, and J.D.C. Little "A Market Share Theorem" Journal of Marketing Research 120 (1975) 136-141

[7] Bischi G.I., Gardini, L. and Kopel, M "Analysis of Global Bifurcations in a Market Share Attraction Model", Journal of Economic Dynamics and Control, 24 (2000) 855-879

[8] Bischi G.I. and L. Gardini "Global Properties of Symmetric Competition Models with Riddling and Blowout Phenomena", Discrete Dynamics in Nature and Society, 5, (2000) 149-160.

[9] Bischi G.I., Gallegati, M. and Naimzada, A. "Symmetry-Breaking bifurcations and representative firm in dynamic duopoly games", Annals of Operations Research, 89 (1999) 253-272.

[10] Bischi G.I. and M. Kopel "Multistability and path dependence in a dynamic brand competition model" Chaos, Solitons \& Fractals, 18 (2003) 561-576

[11] Bischi G.I. and Naimzada A.K. "Global Analysis of a Duopoly game with bounded rationality", in Advances in Dynamic Games and applications, 5, Filar et al. (Eds.), Birkhauser (2000) 361-385.

[12] Bischi G.I., Stefanini, L. and Gardini, L. "Synchronization, intermittency and critical curves in duopoly games", Mathematics and Computers in Simulations, 44 (1998) 559-585.

[13] Bischi G.I. and Gardini, L. "Role of invariant and minimal absorbing areas in chaos synchronization", Physical Review E, 58 (1998) 5710-5719.

[14] Bischi G.I., Chiarella,C., Kopel, M. and Szidarovszky, F. Nonlinear Oligopolies: Stability and Bifurcations, Springer-Verlag, 2010.

[15] Bonanno G., "Oligopoly equilibria when firms have local knowledge of demand", International Economic Review, 29, (1988) 45-55.

[16] Bonanno G. and C. Zeeman, "Limited knowledge of demand and oligopoly equilibria", Journal of Economic Theory, 35 (1985) 276-283.

[17] Buescu J. Exotic Attractors, Birkhauser, Basel, 1997.

[18] Carpenter, G.S., L.G. Cooper, D.M. Hanssens, and D.F. Midgley "Modeling asymmetric competition" Marketing Science 7 ( 1988) 393-412.

[19] Cooper L.G. Competitive maps: The structure underlying asymmetric cross elasticities. Management Science 34 (1988) 707-723.

[20] Cooper L.G. and M. Nakanishi, Market-Share Analysis. Kluwer Academic Publishers, 1988.

[21] Devaney R.L., An Introduction to Chaotic Dynamical Systems, The Benjamin/Cummings Publishing Co., Menlo Park, California, 1987.

[22] Farris, P., Pfeifer, P.E., Nierop, E., Reibstein, D. "When Five is a Crowd in the Market Share Attraction Model: The Dynamic Stability of Competition" Marketing - Journal of Research and Management, 1 (1) (2005) 29-45.

[23] Flam S.D. , "Oligopolistic Competition: from Stability to Chaos", in: F. Gori, L. Geronazzo and M.Galeotti (Eds.) Nonlinear Dynamics in Economics and Social Sciences, Lecture Notes in Economics and Mathematical Systems, 399, 232-237, Springer-Verlag, (1993). 
[24] Föllmer H., "Random economies with many interacting agents", Journal of Mathematical Economics, 1 (1974) 51-62.

[25] Fujisaka, H. and Yamada, T. "Stability theory of synchronized motion in coupled-oscillator systems", Progress of Theoretical Physics, 69 (1) (1983) 32-47.

[26] Gallegati M. and A. Kirman (Eds.), Beyond the Representative Agent, Elgar Pub. Co., 1999

[27] Hasler M. and Maistrenko Yu. "An introduction to the synchronization of chaotic systems: Coupled skew tent maps", IEEE Trans. Circuits Syst., 44 (10) (1997) 856-866.

[28] Jakobson M. "Absolutely continuous invariant measures for one-parameter families of one-dimensional maps". Comm. Math. Phys., 81 (1981) 39-88.

[29] Kirman A. P., "Whom or What Does the Representative Individual Represent?", Jou. of Econ. Perspectives, 6 (1992) 117-136.

[30] Kopel M., "Simple and complex adjustment dynamics in Cournot Duopoly Models". Chaos, Solitons 8 Fractals 7 (1996) 2031-2048.

[31] Kopel M., Bischi G.I., Gardini L. "On new phenomena in dynamic promotional competition models with homogeneous and quasi-homogeneous firms", in Interaction and Market Structure. Essays on Heterogeneity in Economics, D. Delli Gatti, M. Gallegati and A.P. Kirman (Eds.), Lecture Notes in Economics and mathematical Ststems, Springer-Verlag, vol. 484 (2000) 55-87.

[32] Lai Y.C., C. Grebogi and J.A. Yorke, "Riddling bifurcation in chaotic dynamical systems", Phys. Rev. Lett. 77 (1996) 55-58.

[33] Maistrenko, Yu., Maistrenko, V.L., Popovich, A. and Mosekilde, E. "Transverse instability and riddled basins in a system of two coupled logistic maps", Physical Review E, 57 (3) (1998) 2713-2724.

[34] Maistrenko, Yu., Maistrenko, V.L., Popovich, A. and Mosekilde, E. "Role of the Absorbing Area in Chaotic Synchronization", Physical Review Letters, 80 (8) (1998) 1638-1641.

[35] May, R.M. Stability and Complexity in Model Ecosystems, Princeton University Press, 2nd edition (1974)

[36] Milnor J., On the concept of attractor, Commun. Math Phys, 99 (1985) 177-195.

[37] Mira C., L. Gardini, A. Barugola and J.C. Cathala, Chaotic Dynamics in Two-Dimensional Noninvertible Maps, World Scientific, Singapore, 1996.

[38] Nagai, Y. and Y.-C. Lai "Periodic-orbit theory of the blowout bifurcation", Physical Review E, 56 (4) (1997) 4031-4041.

[39] Ott E. and J.C. Sommerer, "Blowout bifurcations: the occurrence of riddled basins", Phys. Lett. A, 188 (1994) 39-47.

[40] Pikovsky A.S. and P. Grassberg, Symmetry breaking bifurcation for coupled chaotic attractors, J. Phys. A: Math. Gen. 24 (1991) 4587-4597.

[41] Pecora L. M. and T. L. Carroll, "Synchronization in chaotic systems," Phys. Rev. Lett. 64 (1990) 821.

[42] Puu T. "Chaos in Duopoly Pricing" Chaos, Solitons \& Fractals, 1 (6) (1991) 573-581.

[43] Sacco P.L., "Adaptive Response and Cournotian Behavior", Economic Notes, 20, 474-496, (1991). 
[44] Schult R.I., D.B. Cramer, F.S. Henyey and J.A. Wright, Symmetric and nonsymmetric coupled logistic maps, Phys. Rev A, 35 (1987) 3115-3118.

[45] Sharkovsky A.N., Yu.L. Maistrenko and E.Yu. Romanenko, Difference Equations and Their Applications, Kluwer Academic Publishers, 1993.

[46] Sterman, J.D., 1989. "Modeling managerial behavior: misperceptions of feedback in a dynamic decision making experiment", Management Science 35, 321-339

[47] Stoker T., Empirical Approaches to the Problem of Aggregation over Individuals, Journal of Economic Literature, XXXI (1993) 1827-1874.

[48] Teocharis, R. "On the Stability of the Cournot Solution of the Oligopoly Problem", Review of Economic Studies, 27 (1960) 133-134.

[49] Thunberg, H. "Periodicity versus Chaos in One-Dimensional Dynamics", SIAM Review, 43 (1), 2001, 3-30.

[50] Turnovsky S.J., Methods of Macroeconomic Dynamics, The MIT Press, 1995.

[51] Varian H.R., Microeconomic Analysis, 3rd edition, W.W. Norton \& Company, 1992.

[52] Venkataramani S.C., B.R. Hunt and E. Ott, Bubbling transition, Phys. Rev. E, 54 (1996) 1346-1360.

[53] Wansink, B., R.J. Kent, and S.J. Hoch "An Anchoring and Adjustment Model of Purchase Quantity Decisions", Journal of Marketing Research 35 (1998) 71-8 . 